\title{
浅析大数据在智能交通系统中的应用
}

\author{
罗超 曾 柯
}

重庆通拓交通规划设计有限公司，重庆 401147

[摘要] 随着城市化建设脚步的加快, 以及小汽车保有量不断增大, 城市交通压力与日俱增。当今科学技术日新月异, 尤其近 年来大数据技术的异军突起, 在城市实施智能交通系统建设中, 通过大数据技术的充分应用, 可有效处理大量采集到的交通 信息, 可较大程度缓解城市交通压力。通常交通大数据十分庞杂，且信息渠道多样，所以通过大数据技术的应用，挖掘交通 数据的潜在价值, 可使得交通数据处理变的高效, 进一步实现城市智能交通管理。因此, 笔者对大数据在智能交通系统中的 应用进行了浅析。

[关键词]大数据; 智能交通; 应用

DOI: $10.33142 /$ sca.v2i6.935

中图分类号: U495;TP311.13

文献标识码：A

\section{Application of Big Data in Intelligent Transportation System}

\author{
LUO Chao, ZENG Ke
}

Chongqing Tongtuo Transportation Planning \& Design Co., Ltd., Chongqing, 401147, China

\begin{abstract}
The urban traffic pressure is increased day by day by the acceleration of urbanization and increasing of car ownership. Nowadays, science and technology are changing rapidly, especially the developing of big data technology in recent years. Application of big data technology can effectively process the traffic information and relieve urban traffic pressure in the construction of intelligent transportation system. Traffic data is complex and the information channels are diverse, so application of big data technology can excavate the potential value of traffic data, make traffic data process efficiently and realize urban intelligent traffic management. Therefore, the author makes the application analysis of big data in intelligent transportation system.
\end{abstract}

Keywords: big data; intelligent transportation; application

\section{引言}

数据是智能交通系统中重要组成部分, 数据的采集与处理是智能交通数据应用的关键环节。大数据技术可在短时 间内收集海量数据并储存，同时高效完成数据信息的处理工作，继而应用到智能交通系统中。通过技术化处理将海量 交通数据可视化，可有效增强大数据应用价值，同时大幅提升智能交通管理水平及实效性。

\section{1 大数据与交通大数据概述}

\section{1 大数据与交通大数据特征}

当今信息技术、互联网技术发展非常迅速，大数据其实在人们的工作、生活中扮演着非常重要得角色。所谓大数据, 简单来说就是海量数据, 其主要特点是数据的存储量大、类型多、处理速度快, 就交通大数据而言, 主要特点如下:

一是数据量庞大, 数据的来源十分广泛, 存储时间也相对较长;

二是要求处理速度非常快, 因交通流实效性较强, 数据必须及时、快速处理;

三是模态多样，数据的来源较广泛，类型也较多，模态显得十分多元;

四是价值非常高，交通数据在时间和空间方面均呈现多元化，故应用价值很高;

五是具有可视化特征, 交通运行可通过可视化方式呈现出来 ${ }^{[1]}$ 。

\section{2 智能交通系统中大数据应用优势}

大数据在智能交通系统中应用优势主要体现以下两点:

一是可大幅提高交通运行效率。交通大数据具有实时性，通过对交通状况展开实时监测，并利用智能化技术对交 通数据实时处理与发布，将交通大数据予以实时应用，交通运行的整体效率得到有效提高。

二是切实保障交通安全。通过对大数据实时采集与分析, 将结果反馈给车辆, 精准诱导车辆安全行驶。比如, 交 通联合探测设备可实现数据无缝收集与传递, 通过构建行车安全模型, 分析车辆运行的轨迹, 再反馈给行驶车辆诱导 驾驶，可主动提高道路交通安全水平 ${ }^{[2]}$ 。 


\section{2 智能交通中的大数据理论}

\section{1 信息资源整合}

信息搜集与大数据分析子系统其实质就是结合用户提出的信息请求, 服务需要利用适当的信息处理系统来对信息 数据实施综合研究, 之后将加工后的信息发送到分支系统, 之后传递到用户手中。这样能够为 ITS 给予准确的信息。 在实施信息数据收集整理工作的时候需要信息收集，网页信息选择，信息数据导入等诸多技术的辅助。其次，ITS 系统 能够完成与其他系统实施信息传递和共享, 能够有效的提升整个系统的运行效率和质量。ITS 系统不但能够高效的对信 息进行收集和分析, 但是在遇到突发事件的时候还是需要利用人工操作来完成信息的收集 ${ }^{[3]}$ 。

\section{2 储存数据处理}

将大数据信息加以统一收集整理并加以存储, 是确保智能化交通系统稳定运行的基础。结合信息数据的种类的差 别, 将信息数据进行分类存储, 这样能够更好的提升信息使用的效率。

智能交通系统内部数据信息种类繁多, 牵涉层面较广, 信息接收时间存在一定的差异, 进而随之也产生了不同类 型的信息处理技术, 我们需要充分的结合实际情况选择适当的计算方法来对信息数据加以处理更好的为 ITS 运行提供 支持。交通大数据处理其实质是针对信息的形式, 内容实施综合的分析研究, 最为能够结合分析结果来对信息数据实 施分类并加以存储, 为后期的使用提供便捷。

\section{3 大数据技术在智能交通中的几点应用}

\section{1 交通诱导中的应用}

交通诱导是通过借助大数据技术, 依据所采集到的数据对当前阶段的交通状态进行合理评测, 再采取短时预测方 式预测交通流量, 同时借助广播、信息情报设备等途径对诱导消息进行传递和散布, 并参考交通流实时改变情况及时 更新诱导方法。同时, 大数据技术可对多种交通检测设备的历史路况数据进行细致有效探析, 归纳出道路交通路况发 展规律, 并与交警平时考勤情况、道路信号改变情况等信息进行有效结合, 可有效地减少交通拥塞, 大幅提升道路服 务水平 ${ }^{[4]}$ 。

\section{2 运输安全中的应用}

城市小汽车保有量在迅速提升, 高峰时段交通冲突严重, 加上天气和周围环境影响, 交通事故的发生很难被有效 控制且难以预测, 如何有效提高交通运输安全性是值得研究的一个问题。将大数据科学合理应用到交通安全分析评估 中，并进行有效交通事件预测，可减少交通事故发生频次，有效避免市民生命财产损失。

\section{3 优化服务中的应用}

服务管理不仅是智能交通系统建设中的重要环节, 也是整个公共交通系统的重要部分。城市中交通工具种类繁多, 尤其是一些提供公共服务的车辆, 例如通勤车、公交车及地铁等, 这种类型交通工具如果不能够保证资源科学合理配 置, 就会严重削减公共交通的服务水平, 降低公交的吸引路。大数据合理使用到公共交通服务中, 将实现资源按需分 配，服务质量亦将显著提高。

\section{4 全息化的交通智能监管}

在社会快速发展的带动下, 现如今已经处在了大数据时代, 使得城市数据资源中心应时而生, 进而也使得城市管 理人员完全摆脱了相互之间连接不顺畅的情况, 为信息数据资源共享创造了良好基础。所有的部门的全部的设备在实 施信息互换以及共享的时候, 需要密切的联系结合社会发展趋势对城市市政交通监督管理机制加以调整。更好的将城 市规划, 通信网络模式, 北斗定位等前沿技术加以综合运用。

\section{5 合理使用信号控制技术}

在社会科学技术水平大幅度提升的影响下, 使得国内通信网络以及信号控制技术得到了明显的进步。首先是是需 要将佳同信息加以搜集, 针对收集工作使用的方法进行不断的优化, 保证其具有良好的实用性, 促使其灵活性加以提 升, 更好的提升车辆的信息识别性能, 这样能够从根本上解决传统程序中存在的问题。其次, 在针对车辆性能实施研 究创新工作的时候, 将智能化理念运用到信号控制系统中, 保证其能够达到需要的智能化水平的基础上, 不仅能够完 成单点控制操作, 并且可以对核心通道以及区域信息实施综合分析和判断。最后是对操作技术实施综合评价, 更好的 
促使民众能够对智能化处理技术加以准确的了解。

\section{6 一站式的交通信息服务}

未来的交通信息服务范畴会逐渐的扩充，不但局限于旅游指导，应急信息公布的处理方面。交通信息服务会提供 大范围的信息基础建设, 并且会对整个地区道路交通情况以及地区交通领域发展趋势实施综合服务, 这样才能更好的 带动整个地区经济的稳定发展, 促进民众生活质量的不断提升, 进而建成一站式的交通服务中心。因为科技的高速发 展与进步, 人工智能会在交通领域取得广泛的用处。

\section{4 大数据在智能交通中应用效果分析}

\section{1 增强交通系统感知力}

物联网是大数据在生活中的主要展现形式, 主要包括感知、网络、应用等三个部分。感知层是物联网技术的基础 层, 可以通过各种传感器获得更加准确、可靠的数据。交通系统中的信息采集多布设在道路两侧、车辆、站台等位置, 通过信息传输装置把信息反馈给总服务器, 服务器对数据信息进行分析处理, 向用户提供所需要的综合交通信息, 将 极大提高出行者的出行效率, 保证交通安全。因此, 大数据应用将极大增强交通系统的感知力 ${ }^{[5]}$ 。

\section{2 实现交通数据实时共享}

通过对大数据实施云计算处理, 可以极大提高数据处理效率和准确度, 实现大数据的实时共享。将数据发布给交 通参与者后, 可制定出最优交通出行路径, 诱导交通需求在路网中均衡分布, 提高路网交通运行效率。

\section{3 有效缓解交通拥堵问题}

大数据与 ITS 的融合是解决城市交通拥堵的重要手段。出行者可以从 ITS 中获得有关道路交通、车辆等方面的实 时信息, 继而从智能系统中选择最优出行方案, 包括出行路线、交通工具等, 如此一来, 可通过 ITS 调配交通分布, 缓解城市交通拥堵。

\section{结语}

在智能交通系统运用成熟之际, 尤其是大数据与智能交通的深度融合, 有效解决了交通数据量不足的问题, 大幅 提高了交通系统的便捷性、高效性、时效性，使得交通管理变的更加科学合理。

\section{[参考文献]}

[1]张溪. 大数据下智能交通系统的发展综述 $[\mathrm{J}]$. 信息与电脑 (理论版), 2019 (01) : 17-19.

[2]付轶欣. “互联网+”时代大数据在智能交通系统的应用研究 [J]. 科技经济导刊, 2018 (01): 27-15.

[3] 罗淑兰, 潘福全, 王昕, 元荣杰, 张丽霞. 大数据在城市交通中的应用研究 [J]. 现代交通技术, 2016,13(05): 76-80.

[4]陈阳.大数据在智能交通系统中的应用研究 [J].信息通信, 2016 (07) : 142-143.

[5]白玲玲, 韩天鹏. 大数据在智能交通系统中的应用研究 [J]. 电脑知识与技术, 2015, 11 (10) : 204-206.

作者简介: 罗超, (1987-) , 男, 四川内江人, 硕士研究生毕业, 从交通规划设计。 